\title{
Linkage Analysis of SPR3 Locus and Pi45(t), and Evaluation of Yield-Related Traits Using Near Isogenic Lines From a Cross Between Japonica Rice
}

\author{
Mark Edward Fabreag ${ }^{1}$, Dong-Min Kim ${ }^{1}$, Ju-Won Kang ${ }^{1}$, Soo-Jin Kwon², Yeo-Tae Yun ${ }^{3}$, Sang-Nag Ahn ${ }^{1 *}$ \\ ${ }^{1}$ Department of Agronomy, College of Agriculture \& Life Sciences, Chungnam National University, Daejeon 305-764, Republic \\ of Korea \\ ${ }^{2}$ National Academy of Agricultural Science, Rural Development Administration, Suwon 441-707, Republic of Korea \\ ${ }^{3}$ Chungnam Agricultural Research and Extension Services, Yesan 340-861, Republic of Korea
}

\begin{abstract}
Although traditional rice varieties and wild rice species exhibit vast genetic diversity, the transfer of useful genes to modern varieties is often hampered by linkage drag. In this study, the previously identified blast resistance locus Pi45(t) from a cross between 'Ilpumbyeo' and 'Moroberekan' was linked to the spreading-type panicle caused by the SPR3 locus. Using InDel4 and RM17579 linked to the Pi45(t) and the $S P R 3$, respectively, the distance between the two loci was estimated to be $6.9 \mathrm{cM}$. This suggests a tight, yet incomplete linkage and provides the opportunity to utilize Pi45(t) in breeding programs without including SPR3. Two groups based on the genotype at the SPR 3 locus were assembled; the CLosed Panicle (CLP) and SPReading panicle (SPR) groups, with lines which were homozygous for the Ilpumbyeo and Moroberekan alleles, respectively. A comparison between the traits of CLP and SPR groups revealed a decrease in 1000-grain weight and length and an increase in spikelets per panicle and secondary branches in the SPR group. This complicates selection against SPR3 as it is not clear whether these quantitative trait loci are linked to either SPR3 or Pi45(t). Re-evaluation of these traits using lines recombinant at the two loci would be necessary to clarify this issue.
\end{abstract}

Keywords Blast resistance, Introgression Lines, Linkage drag, Rice, Spreading type

\section{INTRODUCTION}

The quest to develop better rice (i.e., Oryza sativa and Oryza glaberrima) varieties for sustainable food production is nowhere near its end. There is an ever-increasing demand for food as a result of the booming population. Unfavorable growing environments are hindering efficient rice production. Likewise, rice production is being limited by the depletion of resources, such as irrigation water and arable land. Altogether, these events pose a great threat to food security (Khush 1999 and 2005; Zhang 2007).

To cope up with these challenges, breeders have been using traditional rice varieties, wild rice, and wild relatives of rice as sources of genetic variation to further improve the performance of modern rice varieties. One of the obstacles to this approach however, is that most agronomically important genes are linked with undesirable traits (Ikehashi and Araki 1986). This is also known as the linkage drag problem. Overcoming this is inherently difficult in conventional breeding programs. Nonetheless, with the use of molecular markers and a proper breeding population, efficient introgression of important genes, without the undesirable linked traits, can be achieved (Fukuoka et al. 2009; Collard et al. 2005; Tanksley et al. 1989).

In a previous study, Kim et al. (2011) mapped Pi45(t), a locus conferring durable resistance against rice blast disease, on chromosome 4 using advanced introgression lines (AILs) from a cross between a temperate japonica cultivar 'Ilpumbyeo' and a tropical japonica cultivar 'Moroberekan', the latter being the donor parent. A near isogenic line containing the Pi45(t) quantitative trait loci (QTL) region (NIL-QTL) was then selected from the AILs to further

Received June 10, 2014; Revised June 13, 2014; Accepted June 16, 2014; Published June 30, 2014

*Corresponding author Sang-Nag Ahn, ahnsn@cnu.ac.kr, Tel: +82-42-821-5728, Fax: +82-42-822-2631 
characterize the gene. 'Ilpumbyeo' has a closed-type panicle. The NIL-QTL however, was showing the spreading-type panicle. This is a less desirable panicle type for cultivation purposes. The spreading-type panicle, which is caused by the $S P R 3$ locus, was mapped within the upstream regulatory region of the gene OsLG1 (Eiguchi and Sano 1990; Ishii et al. 2013; Luo et al. 2008; Zhu et al. 2013).

OsLG1 was first described for controlling the development of auricle, ligule, and laminar joint (Lee et al. 2007), but was later shown to affect panicle architecture, too (Eiguchi and Sano 1990; Ishii et al. 2013; Luo et al. 2008; Zhu et al. 2013), suggesting a pleiotropic effect. Panicle architecture has been well studied due to its perceived direct effect on yield. A few of the specific traits related to panicle architecture that affects yield include, but are not limited to, panicle length (PL), spikelet per panicle (SPP), number of primary branches $(\mathrm{PB})$, and number of secondary branches (SB).
The objectives of this study are: i) to break the linkage of Pi45(t) with the SPR 3 locus that causes the spreading-type panicle and ii) to assess whether there is a change in traits affecting yield corresponding to the change from spreadingtype to closed-type panicle.

\section{MATERIALS AND METHODS}

\section{Development of experimental materials}

An 'Ilpumbyeo' NIL-QTL, harboring a 'Moroberekan' chromosome segment of $\sim 4$ Mbp encompassing the Pi45(t) gene region (Fig. 1) on chromosome 4, was developed. 'Ilpumbyeo' has a closed-type panicle, while the NIL-QTL has a spreading-type panicle (Fig. 2). The spreading-type panicle has been reported to be caused by the SPR 3 locus (Eiguchi and Sano 1990; Ishii et al. 2013; Luo et al. 2008; Zhu et al. 2013). To break the linkage between Pi45(t)

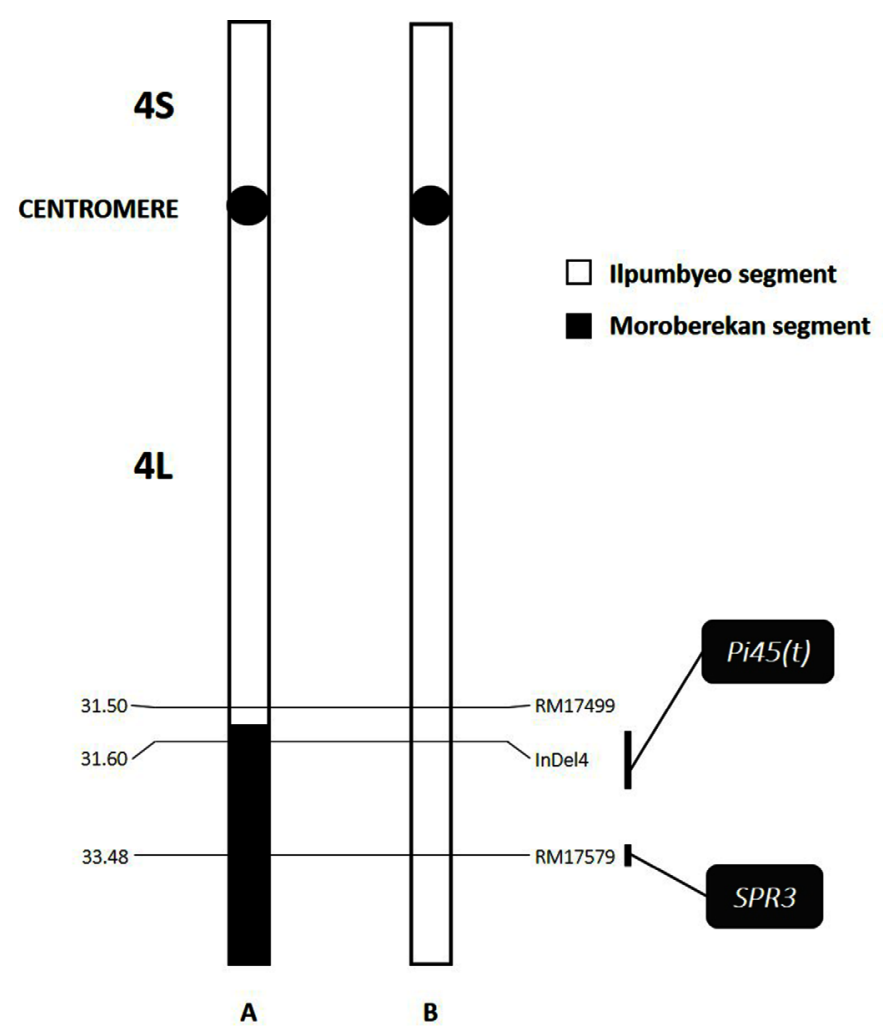

Fig. 1. Graphical representation of chromosome 4 of the (A) NIL-QTL and (B) 'Ilpumbyeo' used in this study, showing the relative locations of Pi45(t) and SPR3, and their corresponding linked markers that were used in this study. No other Moroberekan introgression segment was detected on the other 11 chromosomes. The introgression region starts at approximately $31.5 \mathrm{Mbp}$ down to the telomere. 
and the SPR3 locus, the NIL-QTL was backcrossed to 'Ilpumbyeo'. The subsequent $\mathrm{F}_{2}$ population was used to obtain recombinant plants and to estimate genetic distance between the two loci. Several $F_{2}$ plants were selected and grouped based on their genotype at the Pi45(t) and SPR3 loci. The first group, henceforth referred to as the CLP (CLosed Panicle) group, was composed of $\mathrm{F}_{2}$ plants homozygous for the 'Ilpumbyeo' allele at both loci; the second group, henceforth referred to as the SPR (SPReading panicle) group, was composed of $\mathrm{F}_{2}$ plants homozygous for the 'Moroberekan' allele at both loci. Progenies of the selected $F_{2}$ plants were used to produce $F_{3}$ lines, each line coming from an individual $F_{2}$ plant. These $F_{3}$ lines were then used to evaluate the effect of the SPR 3 locus on yield and other agronomically important traits in rice.

\section{Phenotype evaluation}

A total of $305 \mathrm{~F}_{2}$ plants were cultivated at the experimental field at Chungnam National University, Daejeon, Republic of Korea (CNU) in 2012. Plants were planted with a spacing of $15 \mathrm{~cm} \times 30 \mathrm{~cm}$ (within line $\times$ between lines). For the evaluation of the effect of the SPR 3 locus, 22 $\mathrm{F}_{3}$ lines were cultivated at $\mathrm{CNU}$ and at Chungcheongnamdo Agricultural Research and Extension Services, Yesan, Republic of Korea (ARES) in 2013. Each $F_{3}$ line was planted in two rows with 21 plants per row, following the same spacing as mentioned above. The traits evaluated were plant height $(\mathrm{PH})$, culm length $(\mathrm{CL})$, tiller number $(\mathrm{TN})$, panicle length (PL), number of spikelet per panicle (SPP), number of primary (PB) and secondary branches $(\mathrm{SB})$, panicle neck width (NW), grain length (GL), grain width (GW), grain thickness (GT), 1000-grain weight (TGW), and grain yield per 10 plants (YLD). PH was measured from the plant base to the tip of the highest panicle, while CL was measured from the base to the neck. PL was measured from the neck to the tip of the panicle. TGW was obtained by weighing 250 randomly selected de-hulled seeds for each of the five plants per line. For SPP, $\mathrm{PB}$ and SB, two panicles were evaluated for each of the five plants selected per line. For YLD, ten plants were randomly selected from each line, excluding the first and the last four plants.

\section{Genotyping}

Genomic DNA was extracted from young rice leaves following the CTAB method (Causse et al. 1994). Target regions were amplified using the following PCR cocktail mixture: $5.0 \mu \mathrm{L}(5 \mathrm{ng} / \mu \mathrm{L})$ template DNA, $0.1 \mu \mathrm{L}(5$ units $/ \mu \mathrm{L}) \mathrm{Taq}$ polymerase, $0.8 \mu \mathrm{L}$ dNTP $(2.5 \mathrm{mM}$ each $)$, $1.0 \mu \mathrm{L}(10 \mathrm{pmol}$ each) primer mixture, $2.0 \mu \mathrm{L} \mathrm{10 \times} \mathrm{PCR}$

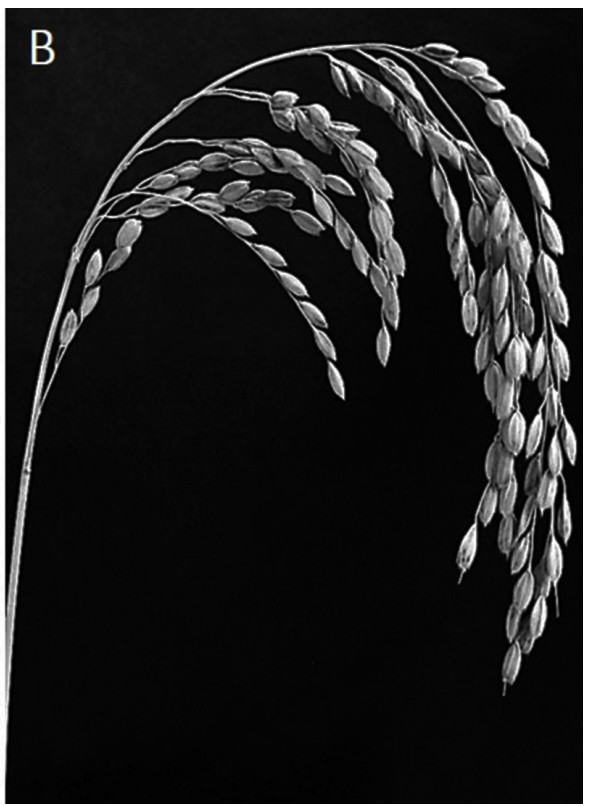

Fig. 2. Panicle of (A) NIL-QTL showing the spreading-type and (B) 'Ilpumbyeo' showing the closed-type. 
buffer (10 mM Tris-HCL, $50 \mathrm{mM} \mathrm{KCL}, 1.5 \mathrm{mM} \mathrm{MgCl}_{2}$, $0.1 \%$ gelatin $\mathrm{pH} 8.3$ ), and $11.1 \mu \mathrm{L}$ triple-distilled $\mathrm{H}_{2} \mathrm{O}$. Using a Thermo Cycler (Bio-Rad), PCR was set under the following conditions: a cycle of denaturation at $94^{\circ} \mathrm{C}$ for 5 min, 35 cycles of denaturation at $94^{\circ} \mathrm{C}$ for $40 \mathrm{sec}$, primer annealing at $56^{\circ} \mathrm{C}$ for $40 \mathrm{sec}$, primer extension at $72^{\circ} \mathrm{C}$ for $1 \mathrm{~min}$, and lastly, a cycle of final extension at $72^{\circ} \mathrm{C}$ for 5 min. Amplicons were resolved using denaturing poly acrylamide gel electrophoresis run for 1-2 $\mathrm{h}$ at 1800-2000 $\mathrm{V}$. Bands were visualized by silver staining (Panaud et al. 1996). InDel4 is a marker linked to Pi45(t) (Kim et al. 2011), while RM17579 is a marker linked to the $S P R 3$ locus (Ishii et al. 2013) (Fig. 1).

\section{Statistical analysis}

All statistical analyses were performed using R (R Core Team 2013). For ANOVA, the genotype at the SPR 3 locus, and therefore the grouping (i.e. CLP and SPR), was considered the treatment, while the $\mathrm{F}_{3}$ lines were considered as replicates.

\section{SPR3 locus sequence comparison}

Previous studies have published the sequences of the respective $S P R 3$ locus region identified. Ishii et al. (2013) sequenced the $9.3-\mathrm{kb}$ upstream region of $O s L G 1$, an accession of $O$. rufipogon, W630, and determined the variants against the Nipponbare (O. sativa ssp. japonica) genome. In a more recent study, Zhu et al. (2013) defined a narrower locus region for $S P R 3$ using a cross between Yuanjiang common wild rice (YJCWR), an accession of $O$. rufipogon, and Teqing (O. sativa ssp. indica). This region of $3.3 \mathrm{~kb}$ was also located upstream $O s L G 1$ and was within the 9.3-kb region defined by Ishii et al. (2013). The SPR3 locus was also reported in CG-14, an O. glaberrima accession (Luo et al. 2008; Fig. 3). It spans around 4.6-kb, and also overlapped the $S P R 3$ regions mentioned above. The sequences of the region for both YJCWR and Teqing were given directly in the paper. The sequence information for CG-14 was not available in the paper.

Whole genome resequencing of 'Ilpumbyeo' and NILQTL were performed following the protocol described by Jeong et al. (2013). Generated paired-end reads were mapped to the 'Nipponbare' genome (RAPDB URL: http://rapdb.dna.affrc.go.jp/) using CLC Assembly Cell. Variants were then called following the Genome Analysis Tool Kit (GATK) best practices protocol using suggested bioinformatics tools such as Samtools (Li et al. 2009), Picardtools (URL: http://picard.sourceforge.net), and GATK (DePristo et al. 2011; McKenna et al. 2010; Van der Auwera et al. 2013). Identified variants were compared with the variants identified in W630, YJCWR, and Teqing.

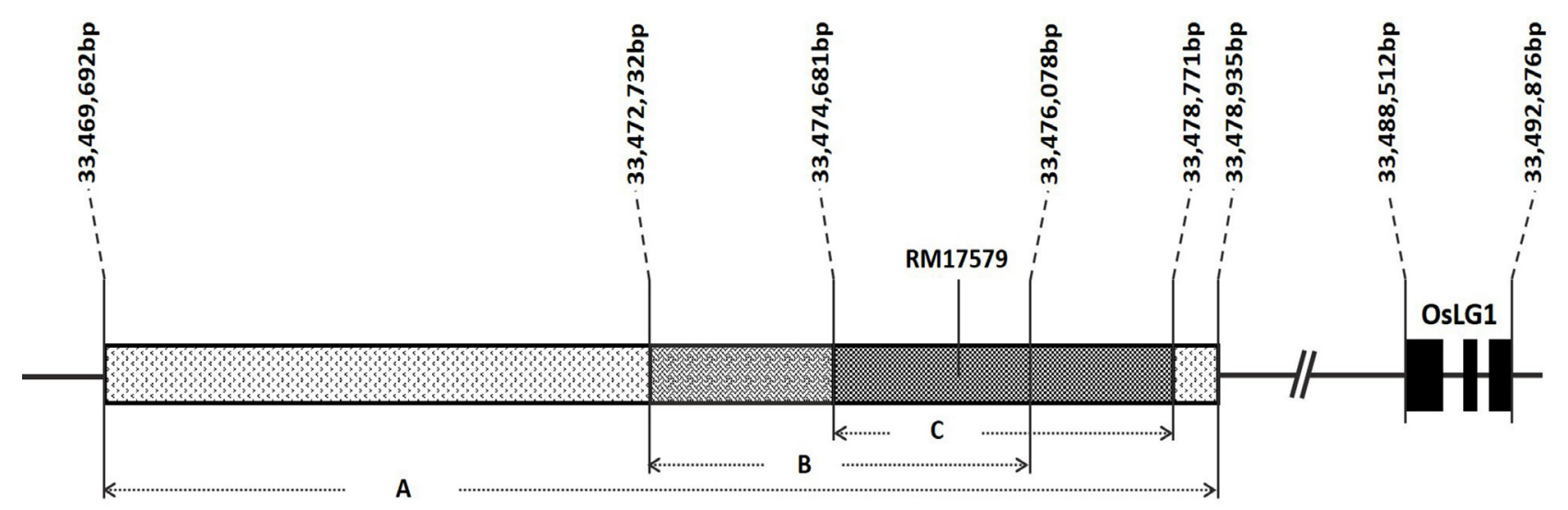

Fig. 3. SPR3 region delimited by (A) Ishii et al. (2013) using W630 (O. rufipogon), (B) Lou et al. (2008) using CG-14 (O. glaberrima), and (C) Zhu et al. (2013) using YJCWR (O. rufipogon). SSR marker RM17579 is located within the intersection of the three regions reported. Positions were based on the Os-Nipponbare-Reference-IRGSP-1.0 reference genome (The Rice Annotation Project Database URL: http://rapdb.dna.affrc.go.jp/). 


\section{RESULTS}

Genetic analysis of SPR3 and linkage between Pi45(t) and SPR3

A total of $305 \mathrm{~F}_{2}$ plants from a backcross between 'Ilpumbyeo' NIL-QTL and 'Ilpumbyeo' were genotyped using two polymorphic markers, InDel4 and RM17579. InDel4 is a marker for an insertion-deletion (thus InDel) identified by sequencing 'Ilpumbyeo' and 'Moroberekan'. RM17579, on the other hand, is an SSR marker that lies within the intersection of the delimited $S P R 3$ region from three different studies (Ishii et al. 2013; Luo et al. 2008; Zhu et al. 2013; Fig. 4). For both markers, alleles segregated following the expected Mendelian ratio of 1:2:1 $\left(\chi^{2}=1.18\right.$, $\mathrm{p}$-value $=0.55 ;$ and $1.27 ; \mathrm{p}$-value $=0.53$ for InDel4 and
RM17579, respectively), suggesting that the alleles of the two loci were inherited normally. Of the $305 \mathrm{~F}_{2}$ plants, only 44 were heterozygous recombinants. From these, the genetic distance was computed as $7.2 \mathrm{cM}$. The physical distance between InDel4 and RM17579 is approximately 1.88 Mbp, based on Os-Nipponbare-Reference-IRGSP-1.0 reference genome (RAPDB URL: http://rapdb.dna.affrc. go.jp/). Assuming $1 \mathrm{cM}$ is equal to $270 \mathrm{kbp}$, this distance between the two markers would translate to $6.9 \mathrm{cM}$, which is relatively close to the genetic distance inferred from the $F_{2}$ population. This confirms that genetic linkage exists between the two loci, although it is incomplete. This result indicates that it is possible to utilize the Pi45(t) gene in breeding programs without the undesirable spreading-type panicle trait.

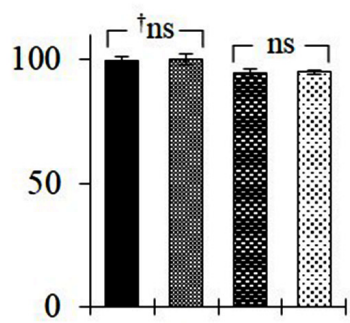

$\mathrm{PH}(\mathrm{cm})$

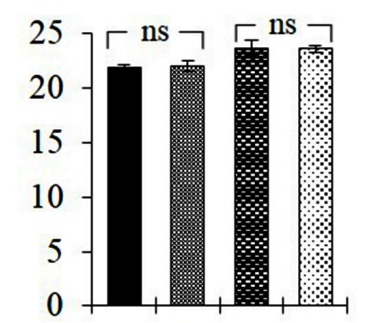

$\mathrm{CL}(\mathrm{cm})$

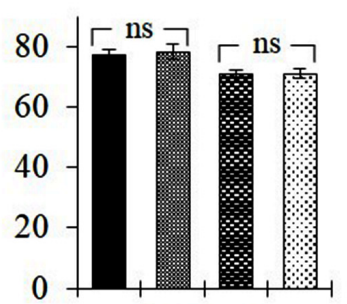

PL (cm)

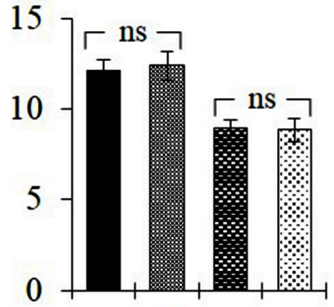

TN (no.)
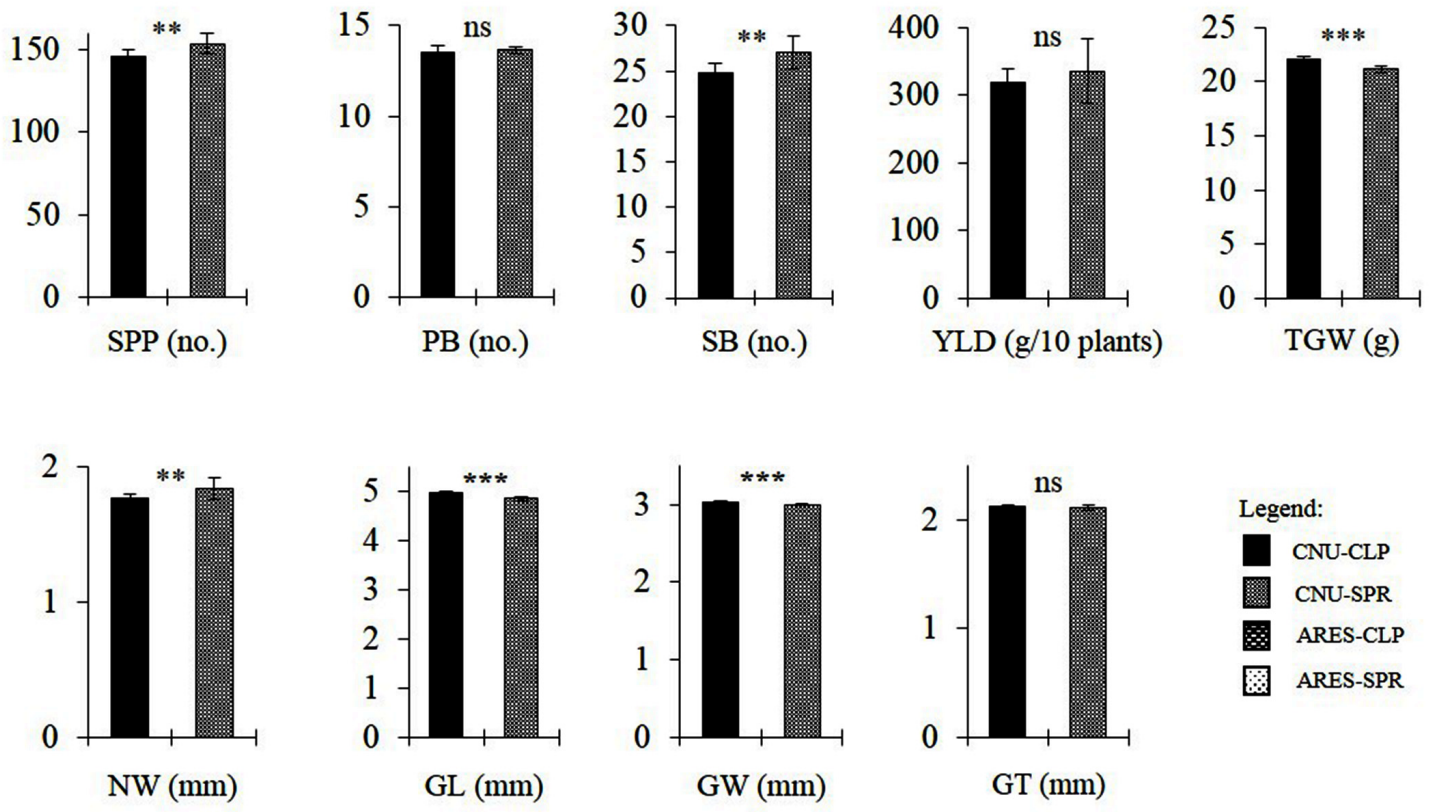

Fig. 4. Trait comparison between the CLP group and SPR group at the two experimental fields; CNU and ARES. Values are means and error bars are $\mathrm{SD}$. $\dagger \mathrm{ns}=$ no significant difference; ‘*', ‘**', ‘***' = significantly different at a $=0.05,0.01$, and 0.001 , respectively. 
In the $F_{2}$ population however, the ratio of spreading type to closed type failed to fit the expected 3:1 ratio $\left(\chi^{2}=27.63\right.$, p-value $=1.47 \mathrm{e}^{07}$ ).

\section{Selection against the SPR3 locus might incur selection penalty}

Two groups of $\mathrm{F}_{3}$ lines were assembled, the CLP group and SPR group, based on the genotype at the SPR 3 locus. However, these lines were non-recombinant between Pi45(t) and SPR 3 loci. Thus, for both loci, the CLP group has the 'Ilpumbyeo' segment while the SPR group has the 'Moroberekan' segment. ANOVA for PH, CL, TN, PL, SPP, PB, SB, NW, GL, GW, GT, TGW, and YLD between the two groups were performed. At $\alpha=0.05$, the CLP group showed significantly lower SPP, SB, and NW, but significantly higher GL, GW, and TGW (Fig. 4). In terms of potential yield, results show the presence of several counteracting loci within the region. Nonetheless, there is a null net effect as shown by the absence of significant difference in YLD. It should also be noted that several traits that were evaluated are dependent on other traits. The significant increase in SPP in the SPR group is most likely caused by the significant increase in SB in the same group. Likewise, significant increases in GL and GW in the CLP group are the most likely causes of the significant increase in TGW. The increase in NW is generally expected to cause an increase in TGW, as it potentially increases translocation capacity. Conversely, the increase in NW in the SPR group was accompanied by a decrease in TGW. This might be due to the sink limit imposed by significantly lower GL and $\mathrm{GW}$ in the SPR group.

\section{Spreading of panicle in Moroberekan caused by a different variation within the $S P R 3$ locus}

The whole genome sequences of both 'Ilpumbyeo' and NIL-QTL were obtained. The average depths of coverage for both region intervals of both parents were generally low ( $\sim 21-23$ reads). Nonetheless, variants of acceptable quality were found (data not shown).

We checked the presence of an SNP which is associated with the change from spreading-type to closed-type panicle and characterized by a nucleotide change from $\mathrm{G}$ (accessions of O. rufipogon) to A located at Chr. 4: 33,477,758; based on the Os-Nipponbare-Reference-IRGSP-1.0 reference genome (RAPDB URL: http://rapdb.dna.affrc.go.jp/). However, based on our data, this SNP was absent on both 'Ilpumbyeo' and NIL-QTL, with the 'Nipponbare' as the reference genome. To check whether this was a false negative, as a consequence of low average depth of coverage, we calculated the number of reads supporting this SNP site. For 'Ilpumbyeo', there were 28 reads, while in NIL-QTL, there were 33 reads supporting the reference allele, both with acceptable QUAL values. The SNP was also absent in the OryzaSNP database (McNally et al. 2009 and 2006, OryzaSNP URL: http://www.oryzasnp.org). These findings suggest that the SNP was indeed absent in 'Moroberekan'. Notably, three SNPs and one InDel between 'Ilpumbyeo' and 'Moroberekan' were found within the 9.3-kb SPR3 region (data not shown).

\section{DISCUSSION}

Linkage drag is a common issue when introducing genes from other sources, including traditional varieties, wild rice, and wild relatives of rice. Here, we showed the linkage drag of the less desirable spreading-type of panicle when the blast resistance gene Pi45(t) from 'Moroberekan' was introgressed to 'Ilpumbyeo'. While it is almost impossible to remove linkage drag between tightly linked loci in conventional breeding, it can be achieved with the use of molecular markers (Collard et al. 2005; Liu et al. 2009; Tanksley et al. 1989). A wide range of molecular markers and marker databases are now publicly available (e.g. RFLP, ESTs, and SSR). Additionally, other markers, such as InDel and SNP (single nucleotide polymorphism) markers, can now be easily designed based on sequencing data, given that the cost of sequencing has decreased. The utilization of resources to address the linkage drag problem was exemplified here. The use of NIL-QTL is a strategy that has been used for fine-mapping QTLs, while at the same time eliminating undesirable traits brought about by linkage drag (Grandillo and Tanksley 2005; Yara et al. 2010). Likewise, the application of this strategy to address linkage drag was demonstrated in this study. 
OsLG1 has been suggested to exhibit pleiotropy, affecting the development of the auricle, ligule, and laminar joint (Lee et al. 2007), as well as panicle architecture (Ishii et al. 2013; Luo et al. 2008; Zhu et al. 2013). Furthermore, a similar SPL (SQUAMOSA-BINDING-LIKE) protein, OSSPL14 was shown to increase panicle branching, and thus grain yield (Miura et al. 2010). This has prompted us to look into other possible functions of OsLG1. Specifically, we evaluated its effect on yield-related traits, as it affects the rice panicle, the main determinant of yield in rice. A similar study was performed by Luo et al. (2008) using an NIL of 'Wuyujing-7' (japonica) having a CG-14 (O. glaberrima) introgression region of 751-kb, which encompasses the SPR3 locus on chromosome 4. Similarly, they found a significant increase in SB and SPP, and a decrease in TGW in the Wuyujing-7 NIL. This NIL has a narrower introgression region than the NIL-QTL used in this present study. Although it is not clear, the similarity of the results in this study with those of the previous study might suggest their linkage to the SPR 3 locus.

Several genes and QTLs for yield components located within the $\sim 4 \mathrm{Mb}$ introgression region have already been identified. Li et al. (1997) identified the QTL $Q G n 4$ for grain number per panicle (SPP). Lin et al. (1996) mapped the QTL tgwt4 for TGW. Both QTLs encompass the OsLG1 region. A more recent study conducted by She et al. (2010) identified the gene flo2 conditioning grain size, grain weight, and grain eating quality. flo2 is located within the delimited region of $\operatorname{tg} w 4$. The allelic relationship among these genes needs to be clarified.

The spreading panicle is a dominant trait (Eiguchi and Sano 1990). In the $F_{2}$ population however, the ratio of spreading type to closed type failed to fit the expected $3: 1$ ratio $\left(\chi^{2}=27.63\right.$, $\mathrm{p}$-value $\left.=1.47 \mathrm{e}^{07}\right)$. This could have been due to phenotyping error, as it was notable that some $F_{2}$ plants were showing an intermediate phenotype: both spreading-type and closed-type panicles were observed on the same plant. It is also noteworthy that the spread panicles in 'Moroberekan' are different from those of O. rufipogon: the latter having a larger angle of panicle branch spreading. The SPR3 locus fine-tunes the expression of OsLG1, the gene controlling panicle architecture (Zhu et al. 2013).

Zhu et al. (2013) narrowed down the SPR3 locus to within a $3.3-\mathrm{kb}$ region. This delimited region was within the 9.3-kb region reported by Ishii et al. (2013), and intersected with the SPR3 region reported by Luo et al. (2008). Furthermore, they were also able to suggest the SNP causing the change from spreading-type to closed-type panicle based on an association analysis (Zhu et al. 2013). This SNP, characterized by a nucleotide change from $\mathrm{G}$ (accessions of $O$. rufipogon) to A, was located at Chr. 4: 33,477,758; based on the Os-Nipponbare-Reference-IRGSP-1.0 reference genome (RAPDB URL: http://rapdb.dna.affrc.go.jp/). This was also present in the W630 used by Ishii et al. (2013). In the association analysis done by Zhu et al. (2013), all of the materials used to represent the spreading-type panicle were actually $O$. rufipogon accessions; all of which shared the same SNP. Neither of the two studies used 'Moroberekan'. We speculate that the spreading-type panicle in 'Moroberekan' is caused by a different variation, but still within the 9.3-kbp regulatory region.

Either the absence of the SNP identified by Zhu et al. (2013) in 'Moroberekan', or the presence of other variants mentioned above, could have caused a lower degree of expression of $O s L G 1$ in this cultivar, resulting in the observed intermediate spreading of the panicles in the $F_{2}$ population. Furthermore, there was no sequence variation between the OsLG1 of 'Ilpumbyeo' and 'Moroberekan', based on our data. These observations further support our speculation that the spreading-type panicle in 'Moroberekan' might either be conditioned by a different variant site within the $9.3-\mathrm{kb}$ regulatory region, or caused by the absence of SNP found by Zhu et al. (2013).

A wealth of genetic diversity can be found in traditional rice varieties, wild rice species, and wild relatives of rice. Though the transfer of useful genes from these sources is usually hindered by linkage drag, this can be addressed through the use of molecular markers and proper breeding materials. The linkage between Pi45(t), a QTL for blast resistance, and the SPR3 locus, which causes spreading panicle in rice, was shown in this study. Nonetheless, with the use of NIL-QTL and appropriate molecular markers, we were able to break this linkage. However, whether or not the selection against the SPR 3 locus would be a clear advantage cannot be ascertained. This issue can be clarified by re-evaluating the traits using lines which are recombinant 
at the Pi45(t) and SPR3 loci. Sequence analysis also revealed that the spreading of the panicle in 'Moroberekan' might be caused by a different variation within the regulatory region of $O s L G 1$, as the SNP previously reported to be associated with the spreading-type panicle was not found in Moroberekan.

\section{ACKNOWLEDGMENT}

This work was carried out with the support of "Cooperative Research Program for Agriculture Science \& Technology Development (Project Title: Next-Generation Biogreen 21 Program, Project No. PJ008136), Rural Development Administration, Republic of Korea.

\section{REFERENCES}

Causse, MA, Fulton TM, Cho YG, Ahn SN, Chunwonse J, Wu K, Xiao J, Yu Z, Ronald PC, Harrington SE, Second G, McCouch SR, Tanksley SD. 1994. Saturated molecular map of the rice genome based on an interspecific backcross population. Genetics 138: 1251-1274.

Collard BCY, Jahufer MZZ, Brouwer JB, Pang ECK. 2005. An introduction to markers, quantitative trait loci (QTL) mapping and marker-assisted selection for crop improvement: The basic concepts. Euphytica 142: 169-196.

DePristo M, Banks E, Poplin R, Garimella K, Maguire J, Hartl C, Philippakis A, del Angel G, Rivas MA, Hanna M, McKenna A, Fennell T, Kernytsky A, Sivachenko A, Cibulskis K, Gabriel S, Altshuler D and Daly M. 2011. A framework for variation discovery and genotyping using next-generation DNA sequencing data. Nature Genet. 43: 491-498.

Eiguchi M and Sano Y. 1990. A gene complex responsible for seed shattering and panicle spreading found in common wild rices. Rice Genet. Newsletter 7: 105-107.

Fukuoka S, Saka N, Koga H, Ono K, Shimizu T, Ebana K, Hayashi N, Takahashi A, Hirochika H, Okuno K and Yano M. 2009. Loss of function of a proline-containing protein confers durable disease resistance in rice. Science 325: 998-1001.

Grandillo S and Tanksley SD. 2005. Advanced backcross
QTL analysis: results and perspectives. In: Tuberosa R, Phillips RL, Gale M (eds.). The wake of the double helix: From the green revolution to the gene revolution. Bologna, Italy: Edizioni Avenue Media: pp. 115-132.

Ikehashi H and Araki H. 1986. Genetics of sterility in rice. In: Rice Genetics. International Rice Research Institute, Los Banos, Philippines, pp. 119-132.

Ishii T, Numaguchi K, Miura K, Yoshida K, Thanh PT, Htun TM, Yamasaki M, Komeda N, Matsumoto T, Terauchi R, Ishikawa R and Ashikari M. 2013. OsLG1 regulates a closed panicle trait in domesticated rice. Nature Genet. 45: 462-467.

Jeong IS, Yoon UH, Ji HS, Lee HJ, Han CD, Hahn JH, An GH and Kim TH. 2013. SNP-based analysis of genetic diversity in anther-derived rice by whole genome sequencing. Rice 6: 6 .

Khush GS. 1999. Green revolution: preparing for the $21^{\text {st }}$ century. Genome 42: 646-655.

Khush GS. 2005. What it will take to feed 5.0 billion rice consumers in 2030. Plant Mol. Biol. 59: 1-6.

Kim DM, Ju HG, Yang P, Han SS, Roh JH and Ahn SN. 2011. Mapping and race specific reaction of the resistance gene Pi45(t) in rice. Korean J. Breeding Sci. 43: 42-49.

Lee J, Park JJ, Kim SL, Yim J and An G. 2007. Mutations in the rice liguleless gene result in a complete loss of the auricle, ligule, and laminar joint. Plant Mol. Biol. 65: 487-499.

Li H, Handsaker B, Wysoker A, Fennell T, Ruan J, Homer N, Marth G, Abecasis G, Durbin R and 1000 Genome Project Data Processing Subgroup. 2009. The sequence alignment/ map (SAM) format and SAM tools. Bioinformatics 25: 2078-2079.

Li Z, Pinson SR, Park WD, Paterson AH and Stansel JW. 1997. Epistasis for three grain yield components in rice (Oryza sativa L.). Genetics 145: 453-465.

Lin HX, Qian HR, Zhuang JY, Lu J, Min SK, Xiong ZM, Huang $\mathrm{N}$ and Zheng KL. 1996. RFLP mapping of QTLs for yield and related characters in rice (Oryza sativa L.). Theor. Appl. Genet. 92: 920-927.

RFLP mapping of QTLs for yield and related characters in rice (Oryza sativa L.). Theor. Appl. Genet. 92: 920-927.

Liu WG, Fan YY, Chen J, Shi YF and Wu JL. 2009. Avoidance of linkage drag between blast resistance gene and the QTL conditioning spikelet fertility based on genotype selection against heading date in rice. Rice Sci. 
16: $21-26$.

Luo JJ, Hao W, Jin J, Gao JP and Lin HX. 2008. Fine mapping of Spr3, a locus for spreading panicle from African cultivated rice (Oryza glaberrima Steud.). Mol. Plant 1: 830-838.

McKenna A, Hanna M, Banks E, Sivachenko A, Cibulskis K, Kernytsky A, Garimella K, Altshuler D, Gabriel S, Daly M and DePristo MA. 2010. The Genome Analysis Toolkit: a MapReduce framework for analyzing next-generation DNA sequencing data. Genome Res. 20: 1297-303.

McNally KL, Childs KL, Bohnert R, Davidson RM, Zhao K, Ulat VJ, Zeller G, Clark RM, Hoen DR, Bureau TE, Stokowski R, Ballinger G, Frazer KA, Cox DR, Padhukasahasram P, Bustamante CD, Weigel D, Mackill DJ, Bruskiewich RM, Rätsch G, Buell R, Leung H and Leach JE. 2009. Genome wide SNP variation reveals relationships among landraces and modern varieties of rice. Proc. Natl. Acad. Sci. USA 106: 12273-12278.

McNally KL, Bruskiewich R, Mackill D, Buell R, Leach JE and Leung H. 2006. Sequencing multiple and diverse rice varieties. Connecting whole-genome variation with phenotypes. Plant Physiol. 141: 26-31.

Miura K, Ikeda M, Matsubara A, Song XJ, Ito M, Asano K, Matsuoka M, Kitano H and Ashikari M. 2010. OsSPL14 promotes panicle branching and higher grain productivity in rice. Nat. Genet. 42: 545-549.

Panaud O, Chen X and McCouch SR. 1996. Development of microsatellite markers and characterization of simple sequence length polymorphism (SSLP) in rice (Oryza sativa L.). Mol. Gen. Genet. 252: 597-607.

Picardtools URL: http://picard.sourceforge.net

R Core Team (2013) R: A language and environment for statistical computing. R Foundation for Statistical Com puting, Vienna, Austria. URL: http://www.R-project.org/. She KC, Kusano H, Koizumi K, Yamakawa H, Hakata M, Imamura T, Fukuda M, Naito N, Tsurumaki Y, Yaeshima M, Tsuge T, Matsumoto K, Kudoh M, Itoh E, Kikuchi S, Kishimoto N, Yazaki J, Ando T, Yano M, Aoyama T, Sasaki T, Satoh H and Shimada H. 2010. A novel factor FLOURY ENDOSPERM2 is involved in regulation of rice grain size and starch quality. The Plant Cell 22: 3280-94.

Tanksley SD, Young ND, Paterson AH and Bonierbale MW. 1989. RFLP mapping in plant breeding: new tools for an old science. Bio/Technology 7: 257-264.

The Rice Annotation Project Database URL: http://rapdb. dna.affrc.go.jp

Van der Auwera GA, Carneiro M, Hartl C, Poplin R, del Angel G, Levy-Moonshine A, Jordan T, Shakir K, Roazen D, Thibault J, Banks E, Garimella K, Altshuler D, Gabriel S and DePristo M. 2013. From FastQ data to high-confidence variant calls: The Genome Analysis Toolkit best practices pipeline. Current Protocols in Bioinformatics 43: 11.10.1-11.10.33.

Yara A, Phi CN, Matsumura M, Yoshimura A and Yasui H. 2010. Development of near-isogenic lines for $B P H 25(t)$ and $B P H 26(t)$, which confer resistance to the brown planthopper, Nilaparvata lugens (Stål.) in indica rice 'ADR52'. Breeding Sci. 60: 639-647.

Zhang Q. 2007. Strategies for developing green super rice. Proc. Natl. Acad. Sci. USA 104: 16402-16409.

Zhu Z, Tan L, Fu Y, Liu F, Cai H, Xie D, Wu F, Wu J, Matsumoto T and Sun C. 2013. Genetic control of inflorescence architecture during rice domestication. Nat. Comm. 4: 2200. 